\title{
Editorial
}

\section{El nuevo paradigma de la salud laboral: alimentación, nutrición y dieta}

\section{Occupational health new paradigm: food, nutrition and diet}

\author{
Liliana Melián-Fleitas' ${ }^{1}$ \\ 1. Servicio Canario de Salud. Atención Primaria de Lanzarote. Lanzarote. España. \\ Recibido: 24-02-2019 \\ Aceptado: 25-02-2019

\section{Correspondencia} \\ Liliana Melián-Fleitas \\ Servicio Canario de Salud. Atención Primaria de Lanzarote. \\ Lanzarote. España. \\ Correo electrónico: lilianamelian@hotmail.es
}

Cada día 3400 de los más de 7400 millones de personas a nivel mundial, acuden a trabajar ${ }^{1}$. El tiempo invertido en el trabajo es mayor que casi cualquier otra actividad, incluyendo dormir. Y se estima que una persona promedio pasará al menos 90.000 horas trabajando durante toda su vida, lo que equivale aproximadamente a más de un tercio de sus horas de vigilia ${ }^{2}$. Estos datos ponen de relieve el impacto que ejerce el trabajo sobre la vida de las personas y por tanto, también sobre la salud y la enfermedad.

Ya Bernardino Ramazzini (1633-1714) ${ }^{3}$, en su libro sobre enfermedades profesionales e higiene industrial, publicado en 1700 con el título "Tratado de las enfermedades de los artesanos», denunció las condiciones insalubres de los mineros, las intoxicaciones con plomo y mercurio en diferentes profesiones, los peligros del sedentarismo y la influencia de los factores ambientales. Recomendaba medidas preventivas que debían abarcar tres niveles: la eliminación del riesgo, su control a través de la ingeniería y la prevención personal.

Ramazzini (considerado el padre de la Medicina del Trabajo) fue un adelantado a su época al reclamar la reducción de la jornada laboral, implementación de descansos, la adopción de posturas correctas (lo que hoy llamamos ergonomía), la adecuada alimentación y la lucha contra la pobreza ${ }^{3}$.

Desde entonces, los programas y estrategias de seguridad y salud laboral se han centrado principalmente en garantizar que el trabajo fuera seguro y que los trabajadores estuvieran protegidos de los daños que del mismo se derivan. Sin embargo, las nuevas tendencias se centran en todos aquellos factores relacionados con el trabajo y que pueden servir para mantener y mejorar la salud y seguridad del trabajador ${ }^{4}$.

Un ejemplo de ello es el National Institute for Occupational Safety and Health (NIOSH), que forma parte de los Centers for Disease Control and Prevention de los EE.UU., que tiene como objetivo concienciar a los empleadores y empoderar a los trabajadores para crear lugares de trabajo seguros y saludables. Una de sus líneas maestras es la "Total Worker Health (TWH)», una «estrategia que integra la seguridad ocupacional y protección de la salud con la promoción de la salud a fin de prevenir lesiones y enfermedades a los trabajadores y mejorar su salud y bienestar». Toma como base lo expuesto anteriormente, al reconocer que el trabajo es un determinante social de la salud y que factores como pueden ser las cargas de trabajo, la exposición al estrés, entornos saludables, programas de actividad física, acceso a alimentos saludables y

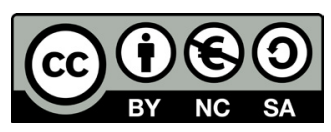

https://creativecommons.org/licenses/by-nc-sa/4.0/ 
licencias remuneradas entre otros, tienen un impacto notable en el bienestar y salud de los trabajadores ${ }^{4,5}$. La evidencia científica actual respalda lo que muchos profesionales de la seguridad y la salud, así como los mismos trabajadores, habían sospechado desde hacía tiempo: los factores de riesgo en el lugar de trabajo pueden contribuir y contribuyen a problemas de salud que habían sido considerados como no relacionados con el trabajó ${ }^{4}$ Por ejemplo, en los trabajadores por turnos y con horarios de trabajo no tradicionales se elevan de forma significativa las tasas de sobrepeso, obesidad y algunas enfermedades metabólicas ${ }^{6,7}$. También hay factores de riesgo vinculados con los trastornos del sueño ${ }^{8}$, las enfermedades cardiovasculares ${ }^{9}$, el estrés, la ansiedad y la depresión entre otras ${ }^{5,10}$.

Los riesgos psicosociales relacionados con el trabajo también presentan efectos negativos para la salud y seguridad de los trabajadores y las empresas, dando como resultado un rendimiento limitado. Y si se mantienen en el tiempo, graves problemas de salud, incluyendo ansiedad, depresión, intentos de suicidio, problemas del sueño, lumbalgias, fatiga crónica, problemas digestivos, enfermedades autoinmunes, enfermedades cardiovasculares, hipertensión arterial y úlceras pépticas. Además de la reducción de la calidad de las relaciones de pareja, familiares y sociales 5 .

Ya en 2007, Díaz Franco ${ }^{11}$ argumentaba que "desde una consideración laboral de la vigilancia de la salud hay un tema desencadenado, directa o indirectamente, por el estrés que, como otros de carácter grave, debe ser recogido entre las prioridades de la medicina del trabajo: se trata de la presencia de hábitos de sobre nutrición generadores de situaciones de sobrepeso y obesidad que constituirían factores de riesgo de patologías múltiples y actuarían como agravantes de cualquier enfermedad».

Estas patologías, además de tener un elevado coste humano, también suponen un elevado gasto a nivel global para empresas y gobiernos. No solo los gastos directos de la atención médica, sino también los costes indirectos de la pérdida de productividad debido al absentismo y al "presentismo", es decir, cuando una persona acude a trabajar pero no puede rendir a plena capacidad debido a un problema de salud físico o mental $^{12}$. De hecho, las pérdidas de productividad debido a las bajas laborales y el presentismo son incluso más elevadas que los costes directos del tratamiento médico (un promedio de 2,30 dólares en pérdida de productividad por cada dólar en gastos médicos) ${ }^{2}$. Datos del Foro Económico Mundial y la Escuela de Salud Pública de Harvard estiman que las principales enfermedades crónicas y las enfermedades mentales se traducirán en un total acumulado proyectado de 47000 billones de dólares en la producción económica perdida a nivel mundial de 2011 a $2030^{2}$.

Una correcta alimentación junto con una adecuada hidratación, tienen el potencial de influir en muchos aspectos del trabajo ${ }^{13}$, sin embargo, resultan escasas las intervenciones nutricionales como medida para mejorar la salud y el rendimiento de los trabajadores. Algo que sería impensable, por ejemplo, en el deporte, donde la nutrición es parte fundamental para optimizar el rendimiento y donde se han desarrollado recomendaciones basadas en la evidencia y las mejores prácticas ${ }^{14,15}$.

Empresas e instituciones tienen la responsabilidad de que los alimentos disponibles en el lugar de trabajo sean nutricionalmente adecuados o por el contrario, hacer que las opciones no saludables no estén disponibles (conforme a la estrategia NAOS) ${ }^{16}$. La industria alimentaria bombardea constantemente al posible comprador con anuncios de productos relacionados con supuestas mejoras en la salud, el estado de alerta, el aumento de energía o la reducción de la fatiga. Y sin un adecuado conocimiento, el trabajador puede tomar malas decisiones nutricionales debido a la falta de información adecuada sobre los beneficios o perjuicios de ciertos productos ${ }^{13}$.

Entre las actuales tendencias centradas en la nutrición y la salud laboral, destaca la creación de un nuevo concepto, la nutra-ergonomía. Definida como «la interfaz entre los trabajadores, su entorno de trabajo y el rendimiento en relación con su estado nutricional. Considera que la nutrición es una parte integral de un lugar de trabajo seguro y productivo que abarca la salud física y mental, así como el bienestar a largo plazo de los trabajadores» ${ }^{13}$.

Desde una perspectiva global, los programas de salud y bienestar en el lugar de trabajo se presentan como la mejor herramienta para afrontar esta problemática creciente ${ }^{12}$. Los programas integrales de salud se definen como «un conjunto de estrategias coordinadas (que incluyen programas, políticas, beneficios, apoyos ambientales y enlaces a la comunidad circundante) que se implementan en el sitio de trabajo, diseñados para mejorar la salud y la seguridad de todos los empleados» ${ }^{17}$. Recientes estudios respaldan la efectividad de estos programas en el lugar de trabajo para mejorar la salud de los empleados y los resultados de productividad $^{13,17,18}$. Además, existe un consenso general en que la combinación de intervenciones de 
múltiples componentes (centradas en el manejo del estilo de vida que abarca el manejo del estrés, la actividad física, la nutrición, el control del hábito tabáquico y el consumo de alcohol) es más efectiva que los programas que se centran en una sola intervención (solo ejercicio por ejemplo) ${ }^{19,20}$.

Esta evidencia apremia a gobiernos, organizaciones científicas y empresas a implementar medidas, políticas y estrategias globales en materia de seguridad y salud laboral que se centren en los factores organizacionales, conductuales y ambientales relacionados con el trabajo y que influyen de forma directa en la salud general del trabajador y de la empresa, prestando especial atención a la nutrición.

\section{REFERENCIAS BIBLIOGRÁFICAS}

1. Banco Mundial. Población activa, total [página Web]. Washington D.C., USA: Grupo Banco Mundial; 2019 [citada 20 enero 2019]. Disponible en: https://bit.ly/2NqeK9V

2. Yeung O, Johnston K. The Future of Wellness at Work [monografía en Internet]. Miami, USA: Global Wellness Institute; 2016 [citada 20 enero 2019]. Disponible en: https://bit.ly/2Nof7S1

3. Ramazzini B. Tratado de las enfermedades de los artesanos (reedición). Madrid, España: Escuela Nacional de Medicina del Trabajo del Instituto de Salud Carlos III; 1999.

4. National Institute for Occupational Safety and Health (NIOSH). Total Worker Health [página Web]. Atlanta, USA: US Centers for Disease Control and Prevention; [actualizada 9 agosto de 2017; citada 20 enero de 2019]. Disponible en: https://bit.ly/2EbIB1x

5. Hassard J, Teoh K, Cox T, Dewe P, Cosmar M, Gründler R, et al. Calculating the cost of work-related stress and psychosocial risks - A Literature Review [monografía en Internet]. Luxembourg: European Agency for Safety and Health at Work - Publications Office of the European Union; 2014 [citada 20 enero 2019]. DOI: 10.2802/20493. Disponible en: https://bit.ly/1LHC74I

6. Schulte PA, Wagner GR, Ostry A, Blanciforti LA, Cutlip RG, Krajnak KM, et al. Work, obesity, and occupational safety and health. Am J Public Health. 2007;97(3):428-36. DOI: 10.2105/AJPH.2006.086900

7. Naug HL, Colson NJ, Kundur A, Santha Kumar A, Tucakovic L, Roberts M, et al. Occupational health and metabolic risk factors: A pilot intervention for transport workers. Int J Occup Med Environ Health. 2016;29(4):573-84. DOI: 10.13075/ijomeh.1896.00570

8. Caruso CC. Negative Impacts of Shiftwork and Long Work Hours. Rehabil Nurs. 2014;39(1):16-25. DOI: 10.1002/rnj.107

9. Veronesi G, Borchini R, Landsbergis P, Iacoviello L, Gianfagna F, Tayoun P, et al. Cardiovascular disease prevention at the workplace: assessing the prognostic value of lifestyle risk factors and job-related conditions. Int J Public Health. 2018;63(6):72332. DOI: $10.1007 / \mathrm{s} 00038-018-1118-2$

10. Wulsin L, Alterman T, Timothy Bushnell P, Li J, Shen R. Prevalence rates for depression by industry: a claims database analysis. Soc Psychiatry Psychiatr Epidemiol. 2014;49(11):1805-21. DOI: 10.1007/s00127-014-0891-3

11. Díaz Franco JJ. Estrés alimentario y salud laboral vs. estrés laboral y alimentación equilibrada. Med Segur Trab. 2007;53(209):93-9.

12. Meyer J, Herrera Cuenca M. Estrategias Globales de Salud en el lugar de Trabajo: Ubicar programas adecuados, factor clave para el éxito. An Venez Nutr. 2013; 26(1):23-25.

13. Shearer J, Graham TE, Skinner TL. Nutra-ergonomics: influence of nutrition on physical employment standards and the health of workers. Appl Physiol Nutr Metab. 2016;41(6 Suppl. 2):S165-74. DOI: 10.1139/apnm-2015-0531.

14. Jäger R, Kerksick CM, Campbell BI, Cribb PJ, Wells SD, Skwiat TM, et al. International Society of Sports Nutrition position stand: protein and exercise. J Int Soc Sports Nutr. 2017;14:20. DOI: 10.1186/s12970-017-0177-8

15. Goldstein ER, Ziegenfuss T, Kalman D, Kreider R, Campbell B, Wilborn C, et al. International society of sports nutrition position stand: caffeine and performance. J Int Soc Sports Nutr. 2010;7(1):5. DOI: 10.1186/1550-2783-7-5

16. Agencia Española de Seguridad Alimentaria. Estrategia NAOS: Estrategia para la nutrición, actividad física y prevención de la obesidad [monografía en Internet]. Madrid, España: Ministerio de Sanida y Consumo, Agencia Española de Seguridad Alimentaria; 2005 [consultada 20 enero 2019]. Disponible en: https://bit.ly/1OfiB1V

17. Lang J, Cluff L, Payne J, Matson-Koffman D, Hampton J. The Centers for Disease Control and Prevention: Findings from the National Healthy Worksite Program. J Occup Environ Med. 2017;59(7):631-41. DOI: 10.1097/JOM.0000000000001045

18. Pelletier K. A review and analysis of the clinical and cost-effectiveness studies of comprehensive health promotion and disease management programs at the worksite: update VIII 2008 to 2010. J Occup Environ Med. 2011;53(11):1310-31.

19. Piepoli MF, Hoes AW, Agewall S, Albus C, Brotons C, Catapano AL, et al. 2016 European Guidelines on cardiovascular disease prevention in clinical practice: the sixth joint task force of the European Society of Cardiology and Other Societies on Cardiovascular Disease Prevention in Clinical Practice (constituted by representatives of 10 societies and by invited experts) Developed with the special contribution of the European Association for Cardiovascular Prevention \& Rehabilitation (EACPR). Eur Heart J. 2016;37(29):2315-81. DOI: 10.1093/eurheartj/ehw106.

20. Schröer S, Haupt J, Pieper C. Evidence-based lifestyle interventions in the workplace - an overview. Occup Med (Lond). 2014;64(1):8-12. DOI: 10.1093/occmed/kqt136

|||||||||||||||||||||||||||||||||||||||||||||||||||||||||||||||||||||||||||||||||||||||||||||||||||||||||||||||||||||||||||||||||||||||||||||||||||||||||||||||||||||||||||||||||||||||||||||||||||||||||||||||||||||||||||||||||||||||||||||||||||||||||||||||||||| | 